\title{
Study of Electric Power Steering System
}

\author{
Shaoyun Zhang \\ School of Physics \& Electronic science \\ Guizhou Normal University \\ Guiyang, China
}

\author{
Yi Wang \\ School of Physics \& Electronic science \\ Guizhou Normal University \\ Guiyang, China
}

\begin{abstract}
Electric power steering system (EPS) has been used to replace traditional hydraulic power steering system (HPS) in automobiles. In an EPS system, the assisting steering power is from an electric motor. In principle, the control of the EPS system mainly involves magnitude and direction's adjustment of motor current to control the torque in order to satisfy steering requirement. This research is aimed at developing steering agility and stability, improving real-time performance of the EPS system. In this paper, the structure and working principle of electric power steering system are analyzed. The dynamic models of torque sensor, input shaft, output shaft, assist motor and linear assist characteristic curves are established. A phase lead compensation control strategy of PID closed-loop control is proposed. The simulation model of the EPS is established based on MATLAB/simulink. The simulation results show that the designed control strategy promotes the real-time performance of the system, and alleviates the contradiction between the steering agility and the sense of road, which prove the validity of the control strategy.
\end{abstract}

Keywords-EPS; assist characteristic; control strategy; phase compensation; simulation

\section{INTRODUCTION}

Steering system is an important part of the automobile and its performance directly affects the driving experience and driving safety. Early automotive steering systems is a pure mechanical steering system, without power-assist system, and the power of steering system is entirely provided by the driver, so that it can not get a good driving experience. Since the 1930s, power-assisted steering system has been developed gradually. Power-assisted steering systems are mainly divided into three types: hydraulic power steering system (HPS), electronically controlled hydraulic power steering system (EHPS) and electric power steering system (EPS $)^{[1]}$. In recent years, the conventional hydraulic steering system has been replaced by electric power steering system, because of its advantages. Compared with the former two kinds, electric power steering system (EPS) has many remarkable advantages. Without hydraulic oil system, the EPS that assist power is provided by the assist motor, greatly reduces the complexity and maintenance costs of steering system and the environmental pollution degree. In addition, it heightens the modularization degree and power-assisted effect of steering system, and improves the fuel efficiency of automobile. Thus it can produce a broad application prospect and economic benefit. According to the different position of the motor, electric power steering systems are divided into four types: steering mast power-assist, rack power-assist, pinion power-assist and double

* Professor Yi WANG is the corresponding author.

Phone: +86085186700647 pinion power-assist. The type of steering mast power and pinion power is used in light vehicle, and the type of double pinion power is used in heavy vehicle. On the constitution, all electric power steering systems have three basic components: electronic control unit (ECU), power-assisted motor and torque sensor mounted on the steering mast ${ }^{[2]}$.

At present, the studies of EPS system mainly focus on advanced control theory, control methods, anti-interference, hardware development, etc.. For the contradiction between the steering agility and the sense of road of steering system, this article establishes the system dynamics models and proposes a control strategy with phase lead compensation, based on analyzing the structure and working principle of electric power steering system.

\section{STRUCTURE AND WORKING PRINCIPLE OF EPS}

The EPS system is mainly composed of steering wheel, torque sensors, electronic control unit (ECU), motor, electromagnetic clutch, retarding mechanism, steering gear and rack. The structure diagram of electric power steering system is shown in figure 1 .

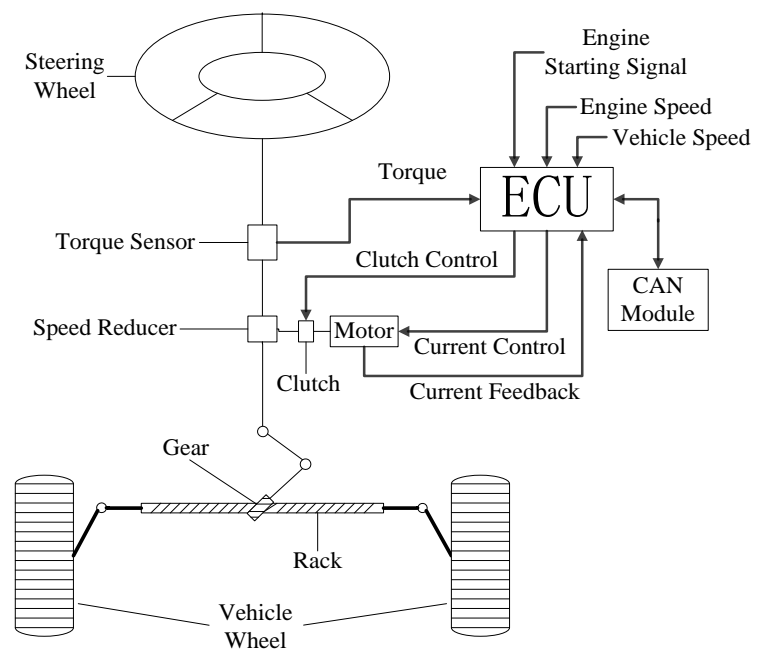

Fig. 1. Electric power steering system structure

After the automobile engine igniting, when the driver turns the steering wheel, the torque sensor installed on the steering shaft measures a steering torque, and sents it to the ECU, and then, according to the torque and speed, the ECU calculates and obtains a optimum current required for the assist motor by the preset assist characteristics curves. At the same time, the ECU collects the signals real-timely, such as input torque, 
speed, voltage, current, rotor position of assist motor, and adjusts the motor current by the preset control strategy, so as to control the output torque and rotation direction of the assist motor, and then the wheels obtain a steering force adapting the running condition by the mechanical transmission mechanism to assist the driver steering in order to gain a better driving experience ${ }^{[3-4]}$. In the whole process of system working, the fault diagnosis module monitors all parts of the system in realtime. Once a failure is detected, the system disconnects clutch immediately, and uses pure manual steering, and gives a fault signal, which ensure the driving safety.

\section{DYNAMIC MODELS OF EPS}

The EPS system model mainly includes torque sensor model, output steering axle model, input steering axle model, assist motor model and steering resistance moment model. According to Newton's law, the following dynamics models of the steering system can be established as follows ${ }^{[5]}$ :

Torque Sensor as in

$$
T_{s}=K_{d}\left(\theta_{m}-\theta_{n}\right)
$$

Input Steering Shaft as in

$$
T_{d}-T_{s}=J_{m} \ddot{\theta}_{m}+B_{m} \dot{\theta}_{m}
$$

Output Steering Shaft as in

$$
T_{a}+T_{s}-T_{r}=J_{n} \ddot{\theta}_{n}+B_{n} \dot{\theta}_{n}
$$

Assist Motor as in

$$
T_{n}-T_{a} / G=J_{a} \ddot{\theta}_{r}+B_{a} \dot{\theta}_{r}
$$

Motor Electromagnetic Torque as in

$$
T_{n}=k_{i} \cdot i_{a}
$$

where $T_{s}$ is the output torque of torque sensor, $T_{d}$ is the input torque of steering wheel, $T_{a}$ is the torque which comes form the assist motor by the retarding mechanism, $T_{r}$ is the resistance torque of output shaft, $T_{n}$ is the electromagnetic torque from the assist motor, $J_{m}$ is the rotational inertia between steering wheel and input steering shaft, $J_{n}$ is the equivalent to the total rotational inertia of output shaft and pinions, $J_{a}$ is the rotational inertia of assist motor, $B_{m}$ is the damping coefficient between steering wheel and input shaft, $B_{a}$ is the damping coefficient of assist motor, $K_{d}$ is the rigidity coefficient of torque sensor, $i_{a}$ is armature current, $\theta_{m}$ is the angle of steering wheel , $\theta_{n}$ is the angle of lower output shaft, $\theta_{r}$ is the angle of assist motor.

In the case of small angle (the angle of tire side-slip is less than $5^{\circ}$ ), the tire characteristics can be considered to be linear, so the resistance torque of steering shaft is described by the following expression ${ }^{[6]}$ :

$$
T_{r}=k_{c} \cdot \theta_{n}
$$

where $k_{c}$ is the steering shaft effective cornering stiffness of front wheels.
Combining (3) (6) can obtain the following function,

$$
T_{s}+G k_{i} i_{a}-k_{c} \theta_{n}=\left(\mathrm{G}^{2} J_{n}+J_{a}\right) \ddot{\theta}_{n}+\left(\mathrm{G}^{2} B_{a}+B_{n}\right) \dot{\theta}_{n}
$$

where $G$ is the transmission ratio between assist motor and steering shaft, $K_{i}$ is the electric torque coefficient of assist motor.

Motor angle as in

$$
\theta_{r}=G \cdot \theta_{n}
$$

Assist Motor Electrical Equation as in

$$
u=R i_{a}+L \dot{i}_{a}+k_{e} \dot{\theta}_{r}
$$

where $u$ is the voltage of motor armature, $R$ is armature resistance, $L$ is armature inductance, $k_{e}$ is anti-electric coefficient assist motor.

\section{CONTROL StRATEGy}

\section{A. Assistance Characteristic Curves}

Assist characteristic of EPS refers to the relationship between input signals (torque and speed) and output signal (assist torque or current of the motor). In the process of driving, the steering resistance decreases with the increase of automobile's speed, so the assist torque of motor should decrease with the increase of speed, and the speed exceeds a certain range, the motor without power. There are three common assist characteristic curves: linear, folding lines and curves type. Linear assist characteristic curves are simple in form, easy to adjust, implement in practice, and used most widely. Therefore, this article takes the linear assist characteristic curves as the research object. Linear assist characteristic can be represented as the following function:

$$
T_{h}=\left\{\begin{array}{cc}
0, & 0 \leq T_{d}<T_{d 0} \\
f(v) \cdot\left(T_{d}-T_{d 0}\right), & T_{d 0} \leq T_{d}<T_{d \max } \\
f(v) \cdot\left(T_{d \max }-T_{d 0}\right), & T_{d} \geq T_{d \max }
\end{array}\right.
$$

where $T_{h}$ is the target torque of motor, $f(v)$ is speed induction coefficient, $T_{h \max }$ is the maximum assist torque of motor, $T_{d 0}$ is the minimum input torque of driver at the beginning of the motor's output power, $T_{d \max }$ is the input torque of driver at the maximum power of motor.

Establishing the characteristic parameters of assist curves as follows ${ }^{[7-9]}$ :

$T_{d 0}=1 \mathrm{Nm}, T_{d \max }=7.6 \mathrm{Nm}, T_{h \text { max }}=22 \mathrm{Nm}$. The speed induction coefficient is determined in accordance with the rules shown in Table 1 (needing to be corrected in real tests). The motor is no power at the speed more than $80 \mathrm{~km} / \mathrm{h}$.

TABLE I. INDUCTION COEFFICIENT OF DIFFERENT SPEED

\begin{tabular}{cccccccc}
\hline Speed $(\mathbf{K m} / \mathbf{h})$ & 0 & 10 & 20 & 30 & 40 & 60 & 80 \\
\hline $\begin{array}{c}\text { Induction Coefficient } \\
f(\boldsymbol{v})\end{array}$ & 3.16 & 2.15 & 1.85 & 1.50 & 1.30 & 1.05 & 0.72 \\
\hline
\end{tabular}


According to the above parameters of table 1, the assist characteristic curves of EPS can be established as shown in figure 2 .

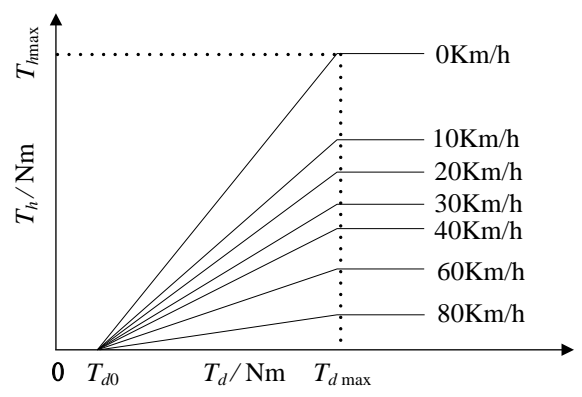

Fig. 2. Assist characteristic curves

The target current of assist motor can be obtained by (11):

$$
i=\frac{T_{h}}{k_{i} \cdot G}
$$

\section{B. Control Algorithm}

EPS control is a current control of motor (magnitude and direction), requiring quick, sensitive and accurate. The stand or fall of EPS control algorithm directly affects the performance of the steering system. Therefore, there must be a reasonable current controller to control the motor current, thereby controlling the output torque of motor. The control algorithm of PID controls the controlled object by adjusting three parameters (proportion, integral and differential) of control errors. PID control, with strong stability and high reliability, is easy to implement and debug, and is suitable for the automobile electric power steering system.

Expression of PID algorithm can be represented as the following formulas:

$$
\begin{gathered}
e(t)=r(t)-y(t) \\
u(t)=K_{p}\left[e(t)+\frac{1}{T_{i}} \int_{0}^{t} e(t) d t+T_{d} \frac{d e(t)}{d t}\right]
\end{gathered}
$$

where $r(t)$ is the target current of assist motor, $y(t)$ is the actual current of assist motor, $u(t)$ is the output signal of PID controller, $T_{i}$ is integral time constant, $T_{d}$ is differentiating time constant.

\section{Phase Compensation}

The controller of EPS, with speed $(V)$ and output of torque sensor $\left(T_{s}\right)$ as input signals, determines the target current of assist motor by the pre-set assist characteristic curves, then controls the motor's power by PWM technology. At the same time, the controller computes the difference between actual current and target current, and adjusts the motor's power through PID arithmetic.

PWM module has time-lag effect. Its transfer function is as follow:

$$
G(s)=1 /\left(T_{s}-1\right), T=(1 / 20000) \mathrm{s}
$$

Besides the delay of PWM, as the inertia and damping of steering system, the torque measured by the torque sensor has a certain hysteresis. These lead to the EPS system has a certain hysteresis, especially in fast turning. In order to enhance the response speed of EPS, it is important that designing a phase compensation module, corrects the torque signal measured by the torque sensor.

Phase compensation module can be described as the follow formula:

$$
G(s)=\left(1+a T_{s}\right) /\left(1+T_{s}\right), a>1
$$

where $a$ is the attenuation coefficient, $T$ is time constant.

The EPS control structure model, with the input signal phase compensation, is established, as shown in figure 3.

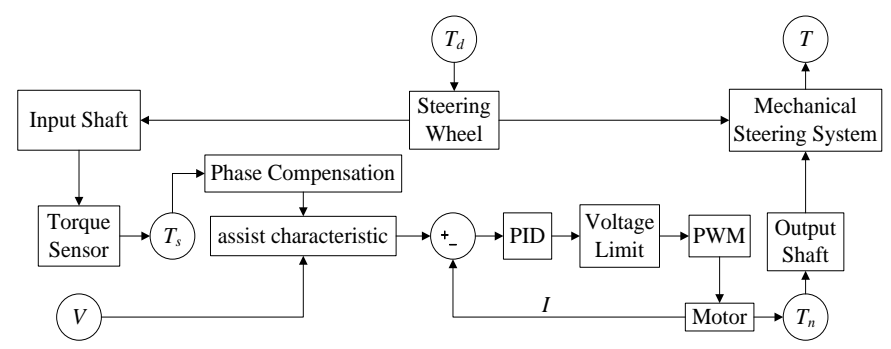

Fig. 3. Control structure model

\section{Simulation and Result Analysis}

\section{A. Establishing Simulation Model of EPS}

EPS system model includes the model of mechanical steering system, motor model and the model of controller. According to the established mathematical model and control structure model, the Simulation Model of EPS can be established based on MATLAB/Simulink, as shown in figure 4.

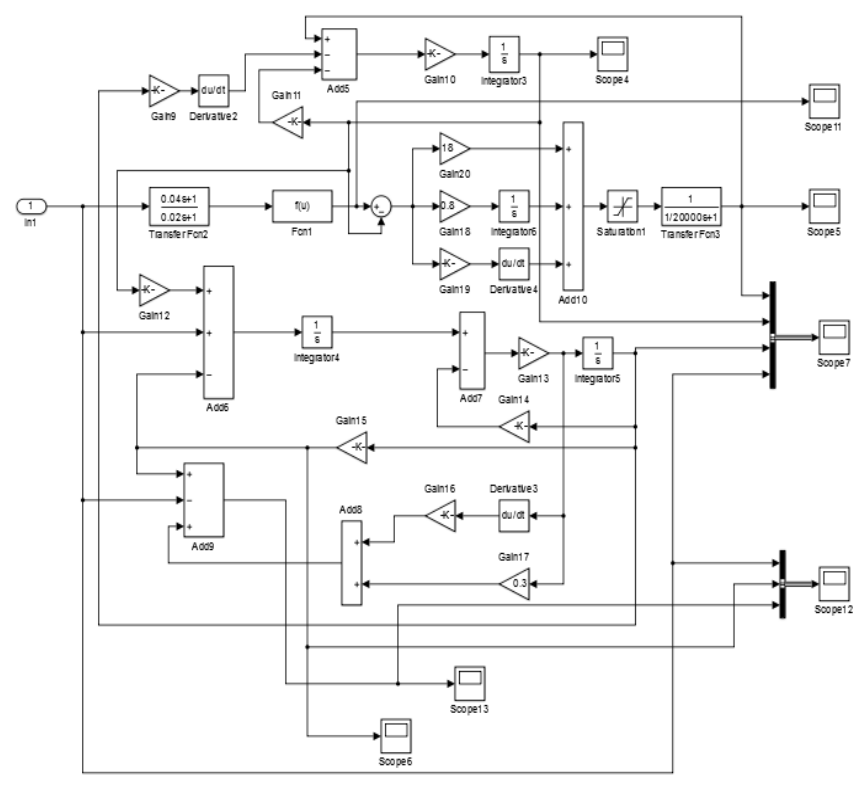

Fig. 4. Simulink simulation model of EPS 


\section{B. Simulation Result Analysis}

According to the Simulink model, combining with the hardware parameters, six groups of simulation experiments are done in MATLAB/Simulink. In order to facilitate observation, respectively taking three kinds of step signal, slope signal and sinusoidal signal do the simulation experiment. The experiment result is shown in figure 5.

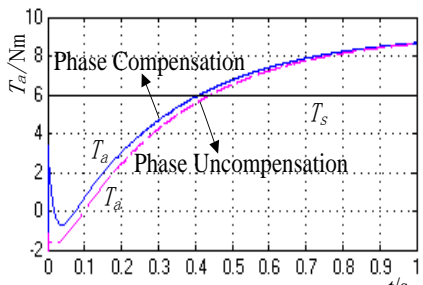

(a) $T_{s}$ for step signal, $V=20 \mathrm{Km} / \mathrm{h} \mathrm{t} / \mathrm{s}$

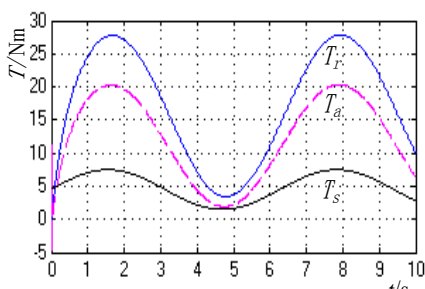

(c) $T_{s}$ for sine signal, $V=0 \mathrm{Km} / \mathrm{h}{ }^{\mathrm{t} / \mathrm{s}}$

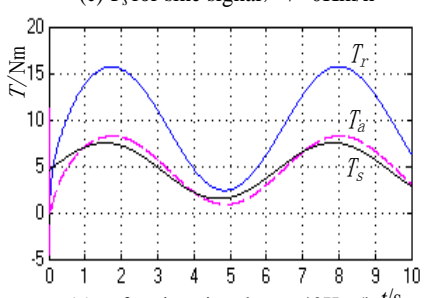

(e) $T_{s}$ for sine signal, $V=40 \mathrm{Km} / \mathrm{h}^{t / \mathrm{s}}$

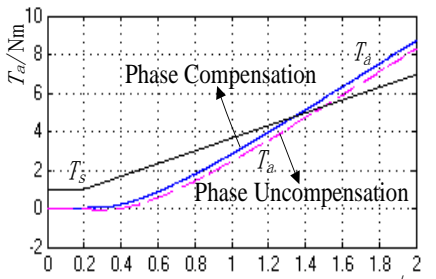

(b) $T_{s}$ for ramp signal, $V=20 \mathrm{Km} / \mathrm{h} t / \mathrm{s}$

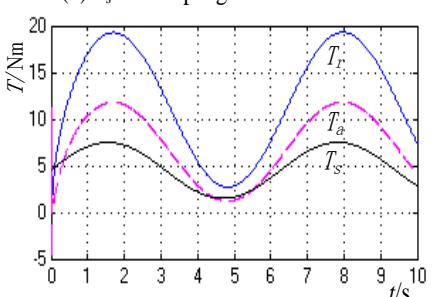

(d) $T_{s}$ for sine signal, $\quad V=20 \mathrm{Km} / \mathrm{h} / \mathrm{s}$

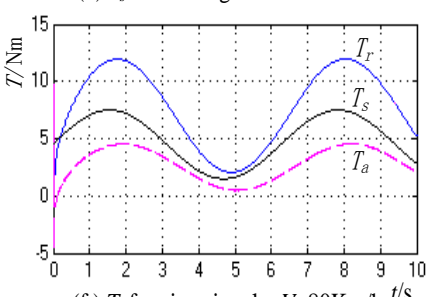

(f ) $T_{s}$ for sine signal, $V=80 \mathrm{Km} / \mathrm{h}$ t/s

Fig. 5. Simulation results of different conditions

In figure $5, V$ for vehicle-speed, $T_{s}$ for input torque of controller, $T_{a}$ for out torque of assist motor, $T_{r}$ for steering resisting torque.

Figure 5(a) is the output responses of assist torque $\left(T_{a}\right)$, when the input torque $\left(T_{s}\right)$ is a step signal. Figure $5(\mathrm{~b})$ is the output responses of assist torque, when the input torque is a ramp signal. Figures 5(a) and (b) show that the output curve based on the input signal phase-compensated is better than the curve under uncompensated input signal, when the input signal changes rapidly. These indicate that in the fast turning, adding phase advance compensation segment, can enhance the response speed of the system and improve the control effect.

Figure 5(c) (f) are the output responses of assist torque $\left(T_{a}\right)$ and steering resisting torque $\left(T_{r}\right)$ in different speeds, when the input torque $\left(T_{s}\right)$ is a sinusoidal signal. It can be seen that the driver's steering torque $\left(T_{d} / T_{s}\right)$ is much smaller than the steering resistance torque $\left(T_{r}\right)$ and with the increase of vehicle speed, $T_{r}$ is gradually reduced and $T_{a}$ is also gradually reduced, and $T_{a}$ can follow the change of the input torque $\left(T_{s}\right)$ in time. Thus, in the driving process, it can be ensured that steering is agile at low vehicle-speed and a good road sense of steering is still keep at high vehicle-speed.

Integrating the whole simulation experiment results, the design of the EPS control strategy can make the output of the controller follow the input well, and improve the response speed of the system after adding the phase advance compensation of input signal. It can obtain the effect of agile steering and good road sense.

\section{CONCLUSION}

In view of the characteristics that low vehicle-speed steering is not enough agile and the road sense of high vehiclespeed is bad, this paper researched the system dynamics models, and designed a linear assist characteristic curves and a control strategy with phase lead compensation, and verified the design in Simulink. The experiment results show that the control strategy designed obtain the effect of agile steering and good road sense, and improves the system real-time performance. The optimization of control performance, will be implemented in the further research of EPS.

\section{ACKNOWLEDGMENT}

This work was supported by International Science \& Technology Cooperation Research Foundation of Guizhou Province (Grant No. [2014]7007), in part by Key Laboratory of Education Department of Guizhou Province (Grant No. KY word[2014] 213).

\section{REFERENCES}

[1] Xiaolin Zhang and Jiming Ma, "Design and Simulation of Electric Power Steering System," Computer Simulation. Beijing, vol. 31, pp. 175-178, March 2014.

[2] Liang Xiong, Heping Liu and Donglin Peng, "A Control Strategy for Automotive Electric Power Steering System Without Torque Senso," Automotive Engineering. Beijing, vol. 35, pp. 711-715, August 2013.

[3] Yaqin Qiu and Xiangwen Zhang, "Sliding Mode Control of Electric Power Steering Control System,” Computer Simulation. Beijing, vol. 30, pp. 159-163, August 2013

[4] Zegang He, Rongwei Shen, Kang Xie and Lina Feng, "Research on control strategy of the vehicle electric power steering system based on brushless DC motor," Modern Manufacturing Engineering. Beijing, pp. 57-61, February 2015.

[5] Dan Xiang, Yongbin Chi, Wubo LI and Yong Yang, "Study on Control Strategy and Simulation for Electric Power Steering System," Control Engineering of China. Shenyang, vol. 20, pp. 254-258, March 2013.

[6] Zhao Liuqi and Jin Dafeng, Fundamentals of Vehicle Dynamics Vehicle Dynamics. Bejing, Tsinghua University Press, 2006.

[7] Toshihide Satake, Masahiko Kurishige, Noriyuki Inoue, Katsuya Ikemoto, Takayuki Kifuku and Kazumichi Tsutsumi, Evaluation of EPS Control Strategy Using Driving Simulator for EPS, SAE Technical paper. dol:10.4271/2003-01-0582, March 2003.

[8] Masahiko Kurishige, Shunichi Wada, Takayuki Kifuku, Noriyuki Inoue, Ryoji Nishiyama and Shigeki Otagaki, A New EPS Control Strategy to Improve Steering Wheel Returnability. SAE Technical Paper, dol: 10.4271/2000-01-0815, March, 2000.

[9] Yang Shu-song, Study on Control of Electric Power Steering System. Luoyang, University of Science and Technology. Luoyang Henan, pp. 22-25, 2011. 